Revista Colombiana de Obstetricia y Ginecología Vol. 59 No. 3 • 2008 • (199-205)

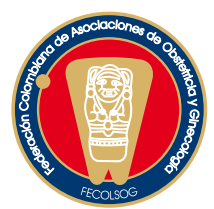

INVESTIGACIÓN ORIGINAL

\title{
EVALUACIÓN DE UNA PRUEBA ELISA \\ IgG DE AVIDEZ PARA TOXOPLASMA PARA \\ EL DIAGNÓSTICO EN EL EMBARAZO \\ Y CORRELACIÓN CON IgM E IgA EN \\ EL LABORATORIO DEL CENTRO DE \\ INVESTIGACIONES BIOMÉDICAS DE LA \\ UNIVERSIDAD DEL QUINDÍO, 2008
}

\section{Evaluating a Toxoplasma IgG avidity ELISA test \\ for dliagnostic purposes during pregnancy and correlating it with Toxoplasma IgM and IgA in the Biomedical Research Centre's laboratory at the Universidad del Quindío, 2008}

Elizabeth Torres-Morales, Bacterióloga*, Jorge Enrique Gómez-Marín, M.D., PhD**

Recibido: febrero 5/08 - Aceptado: julio 29/08

\section{RESUMEN}

Objetivos: establecer el punto de corte de una prueba ELISA IgG de avidez casera en el Centro de Investigaciones Biomédicas de la Universidad del Quindío y correlacionar sus resultados con la fecha de gestación y resultados de IgM e IgA en sueros de pacientes gestantes, analizadas en el laboratorio para establecer el perfil de uso de la prueba.

Materiales y métodos: se estudiaron sueros de 17 pacientes con fechas de infección conocidas, para definir la curva receptor operador (ROC) por

\footnotetext{
* Grupo de Estudio en Parasitología Molecular (GEPAMOL). Centro de Investigaciones Biomédicas, Universidad del Quindío. Armenia, Colombia.

** Centro de Investigaciones Biomédicas, Universidad del Quindío. Armenia, Colombia. Correo electrónico: gepamol2@uniquindío.edu.co
}

la prueba ELISA IgG de avidez anti-Toxoplasma y 91 sueros de pacientes embarazadas con resultados de pruebas IgM e IgA anti-Toxoplasma analizadas en el mismo laboratorio.

Resultados: obtuvimos un punto de corte de avidez menor a 30\%, lo que permite una sensibilidad del $100 \%$ y una especificidad del $88 \%$ para definir si la infección tenía menos de 4 meses de adquirida. En el segundo grupo, de 91 pacientes, encontramos que el 63\% tenía menos de 16 semanas de gestación al momento de la prueba y 3 pacientes tuvieron índices de avidez bajo (<30\%). Se observa que a la mayoría de pacientes se les solicitó la prueba antes de la semana 16 de gestación, lo cual es un indicador de buen uso de la prueba.

Conclusiones: un punto de corte de 30\% para la prueba ELISA de avidez anti-Toxoplasma casera 
en el Centro de Investigaciones Biomédicas de la Universidad del Quindío, identifica infecciones de menos de 4 meses de adquirida.

Palabras clave: toxoplasmosis, IgG de avidez, curva ROC, sensibilidad, especificidad.

\section{SUMMARY}

Aims: determining the cut-off value for a homebased ELISA assay for IgG avidity at the Biomedical Research Centre at the Universidad del Quindío and correlating the results from pregnant females' serum samples with IgM and IgA test results.

Material and methods: 17 patients having known infection dates were studied to determine the receptor operator curve (ROC) for a home-made avidity assay. 91 sera samples from pregnant females were also analysed at the same laboratory for IgM and IgA anti-Toxoplasma antibodies.

Results: $100 \%$ sensitivity and $88 \%$ specificity were obtained by using a $30 \%$ avidity cut-off value for identifying infections acquired less than 4 months beforehand. 63\% of the 91 pregnant females had less than 16 weeks' gestation at the time of examination and three of the patients had low avidity tests (<30\%). Only one case had avidity less than $<30 \%$ after 8 months' infection. The avidity test was requested before the 16th week of the gestational period for most of the patients.

Conclusions: a 30\% cut-off identified sera containing infections having less than 4 months' evolution.

Key words: toxoplasmosis, avidity test, ROC curve, sensitivity, specificity.

\section{INTRODUCCIÓN}

Toxoplasma gondii es un parásito intracelular obligado cosmopolita que causa infección en humanos por zoonosis o por ingestión de alimentos contaminados y, en pocos casos, por transplantes de órganos. ${ }^{1}$ En la mayoría de pacientes la enfermedad es asintomática pero puede causar grandes complicaciones en individuos inmunosuprimidos o inmunodeficientes, llevando a la encefalitis toxoplásmica. ${ }^{1}$ Otro grave problema es la toxoplasmosis congénita, que ocurre cuando la mujer adquiere una infección durante la gestación. La infección aguda en embarazadas no se evidencia en alrededor del $90 \%$ de los casos. ${ }^{2-5}$ En general los signos y síntomas de infección aguda son tan insignificantes que no se resaltan en la historia clínica o no se recuerdan por la gestante. Cuando la gestante es inmunosuprimida por VIH, linfomas o corticoterapia, la transmisión congénita puede ocurrir aún cuando tenga historia pasada de infección por Toxoplasma gondii y presente un perfil serológico de infección crónica. ${ }^{3}$

A pesar de la existencia de marcadores de infección aguda como IgM o IgA, la persistencia de ellos hasta por dos años, luego de ocurrida la infección, hace que la interpretación y evaluación del riesgo de transmisión durante el embarazo sea incierta. ${ }^{3}$ Una alternativa es medir la avidez funcional del antígeno y el anticuerpo, lo que permite estimar el tiempo de adquisición de la infección. La prueba de anticuerpos IgG de avidez ${ }^{6}$ se basa en la distinta fuerza o afinidad que existe en la unión antígeno-anticuerpo. La técnica de avidez de IgG antitoxoplásmica permite diferenciar una infección aguda o primaria de una crónica. En los primeros meses de una infección, el hospedero responde con la producción de anticuerpos de la clase IgG de baja afinidad, la cual aumenta a medida que transcurre el tiempo de infección.

El análisis de la estabilidad de la unión del complejo antígeno-anticuerpo se puede hacer a través de una adaptación de la prueba ELISA IgG para medir la avidez del anticuerpo. Se basa en que los anticuerpos producidos durante la fase aguda de la infección tienen una menor avidez. ${ }^{7}$ La ruptura de la unión entre el antígeno y el anticuerpo, en el caso de los anticuerpos de baja avidez, se puede realizar fácilmente con un agente desnaturalizante como la urea. En este estudio buscamos establecer una curva de madurez de avidez, según el tiempo de adquisición de la infección, en sueros con fecha de inicio de síntomas conocidos, para una prueba ELISA IgG casera de avidez para Toxoplasma en el Centro de 
Investigaciones Biomédicas de la Universidad del Quindío. De otra parte, buscamos examinar la edad gestacional en la cual se está utilizando la prueba y su correlación con los resultados de esta $\operatorname{IgG}$ de avidez con la IgM e IgA anti-Toxoplasma.

\section{MATERIALES Y MÉTODOS}

Diseño: estudio de corte transversal, estandarización de prueba diagnóstica.

\section{Población (pacientes y sueros)}

Utilizamos un primer grupo de sueros de 17 pacientes con fechas conocidas de infección y síntomas de toxoplasmosis ganglionar (diagnosticados en la consulta especializada del centro de salud de la Universidad del Quindío) con el fin de definir una curva de evolución del porcentaje de avidez de la prueba IgG anti-Toxoplasma. La hipótesis de asociación se realizó entre el número de meses desde la fecha estimada de infección (por signos y síntomas clínicos obtenidos durante la elaboración de la historia clínica) y los resultados del porcentaje de avidez de la prueba ELISA para IgG anti-Toxoplasma. La prueba de avidez busca determinar el tiempo de evolución de la infección y discriminar entre dos grupos de pacientes: aquellos con menos de 4 meses de adquirida la infección y aquellos con más de 4 meses de adquirida la infección.

Un segundo grupo analizado fue el de los sueros de 91 pacientes embarazadas atendidas en el programa de control de embarazo de la ciudad de Armenia y Calarcá (estratos socioeconómicos según SISBEN de 1 a 3) y sueros remitidos de Bucaramanga (estratos socioeconómicos según SISBEN de 3 a 6) con edad gestacional conocida; con el cual se buscó determinar a qué momento de la gestación se estaban solicitando las pruebas de avidez. La selección fue por muestreo secuencial de conveniencia. Se excluyeron los sueros de gestantes en los cuales no se contaba con los datos de las semanas de gestación.

\section{ELISA para IgG}

Se utilizó la técnica HUMAN ELISA TOXO IgG (Alemania). En la primera etapa de incubación, los anticuerpos anti-Toxoplasma contenidos en la muestra y en el control suministrado en el estuche, se fijaron específicamente a los antígenos inmovilizados. Al final de la incubación, los componentes excesivos fueron eliminados con solución de lavado. Se añadió posteriormente un conjugado anti $\operatorname{IgG}$ (anticuerpo-anti IgG humana, marcado con peroxidasa) que se fijó específicamente a los anticuerpos IgG, formándose inmunocomplejos típicos. Después de eliminar el conjugado excesivo con la solución de lavado, se añadió el substrato TMB, el cual forma un color azul que se transforma en amarillo después de parar la reacción. La intensidad de este color es directamente proporcional a la cantidad de anticuerpo anti-TOXO IgG en la muestra. La lectura se realizó en un lector de ELISA Dynatech (USA) MR5000 a $450 \mathrm{~nm}$ y con filtro de referencia a $630 \mathrm{~nm}$.

Los resultados se expresaron en unidades internacionales por mililitro (UI/ml). Los sueros de un mismo paciente se analizaron simultáneamente y fueron diluidos si anteriormente habían sido mayor o igual a $300 \mathrm{UI} / \mathrm{ml}$ (límite de cuantificación de la prueba). Cuando los sueros presentaron resultados de densidades ópticas mayores a 3,00 se realizó dilución 1:100.

\section{ELISA para IgG de avidez}

Se siguió una técnica modificada, descrita por Peyron, et al. ${ }^{8}$ Se utilizó el estuche comercial de la marca HUMAN ELISA TOXO IgG para la avidez; cada suero se hizo en duplicado. En la primera etapa del lavado uno de los pozos se lavó 3 veces durante 5 minutos con $300 \mu \mathrm{l}$ de urea 6 molar por lavado. El otro pozo se lavó 5 veces durante 30 segundos con tampón de lavado que suministra el estuche. La urea se prepara con la solución tampón de lavado del estuche. Los resultados se expresaron en porcentaje de avidez ${ }^{9}$ así:

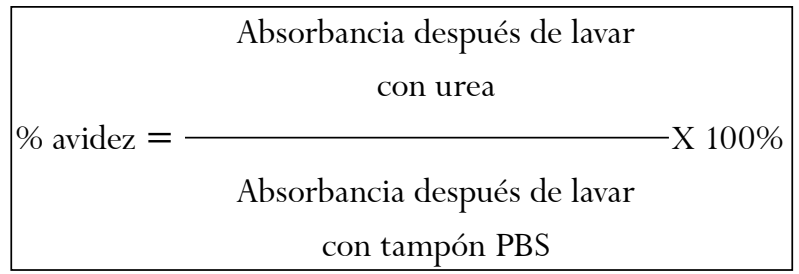




\section{ELISA para IgM, IgA}

Para el análisis de los anticuerpos IgM se utilizó el estuche comercial de HUMAN (Alemania) y técnica de imnunoensayo enzimático de captura para la determinación de anticuerpos IgA de DIAPRO (Italia). Los resultados se expresaron en absorbancias (unidades de densidad óptica obtenidas al leer las muestras en el espectrofotómetro).

\section{Análisis estadístico}

Los datos y gráficas se organizaron en Excel y el análisis estadístico se realizó con el programa SPSS versión 14 (SPSS Inc. Chicago, USA). Se probó la normalidad de distribución de los datos con el test de Kolmogorov Smirnov y luego se realizó un análisis de correlación entre los meses de infección y el porcentaje de avidez. Se calculó la sensibilidad y especificidad de diferentes porcentajes de avidez para determinar si la infección tenía menos de 4 meses de adquirida y se realizó una curva operador receptor (ROC). Para esta evaluación no hubo prueba de oro y los verdaderos positivos (pacientes con menos de 4 meses de infección) y verdaderos negativos (pacientes con más de 4 meses de infección) fueron establecidos de acuerdo con la fecha de inicio de los síntomas. La edad gestacional se estableció de acuerdo con las semanas de amenorrea, el índice de avidez se estableció en porcentaje y los valores de IgA e IgM en valores de absorbancia.

\section{RESULTADOS}

\section{Establecimiento de punto de corte de acuerdo a curva ROC}

El grupo I constituido por 17 pacientes con toxoplasmosis ganglionar fue dividido en 2 subgrupos; el primero con 8 pacientes menores a 4 meses de infección y el segundo con 9 pacientes mayores a 4 meses de infección. A todos los pacientes se les realizó IgG, IgM, IgA e IgG de avidez. En los pacientes con menos de 4 meses de infección, el título de $\operatorname{IgG}$ estuvo comprendido entre 98,5 y >300 UI/ml, el índice de absorbancia de IgM entre 0,96 y 3 ; y el porcentaje de $\operatorname{IgG}$ de avidez entre $8,7 \%$ a $26,3 \%$.

En los pacientes mayores a 4 meses de infección, el título de IgG estuvo comprendido entre 181,5 y $>300 \mathrm{UI} / \mathrm{ml}$, el índice de absorbancia de IgM entre 0,380 y 1,37; y el porcentaje de IgG de avidez entre $24,9 \%$ a $75,7 \%$. La mediana del porcentaje de avidez para los pacientes con menos de 4 meses de infección fue de 21 (rango 8,7-26) y en la de los pacientes con más de 4 meses de infección fue de 55 (rango 24-75). Como la prueba de Kolmogorov Smirnov mostró que los datos de meses de infección no tenían una distribución paramétrica, se calculó el coeficiente de correlación de Spearman entre la duración en meses de la infección y el porcentaje de avidez obtenido por la prueba ELISA para la avidez de IgG anti-Toxoplasma. El porcentaje de correlación de Spearman fue de 0,87 p $=0,000$ (figura 1). Al realizar la curva ROC con sueros de pacientes colombianos y fechas conocidas de infección, se encontró que teniendo como punto de corte una avidez menor a 30\%, se tiene una sensibilidad del 100\% (IC95\% 100-100) y una especificidad del 88\% (IC95\% 68-100) para identificar infecciones adquiridas menores a 4 meses (figura 2). Se compararon varias marcas de urea, sólo se obtuvo una buena correlación de porcentajes de avidez cuando

Figura 1: Pacientes con fechas conocidas de inicio de síntomas de toxoplasmosis ganglionar y los respectivos porcentajes de avidez.

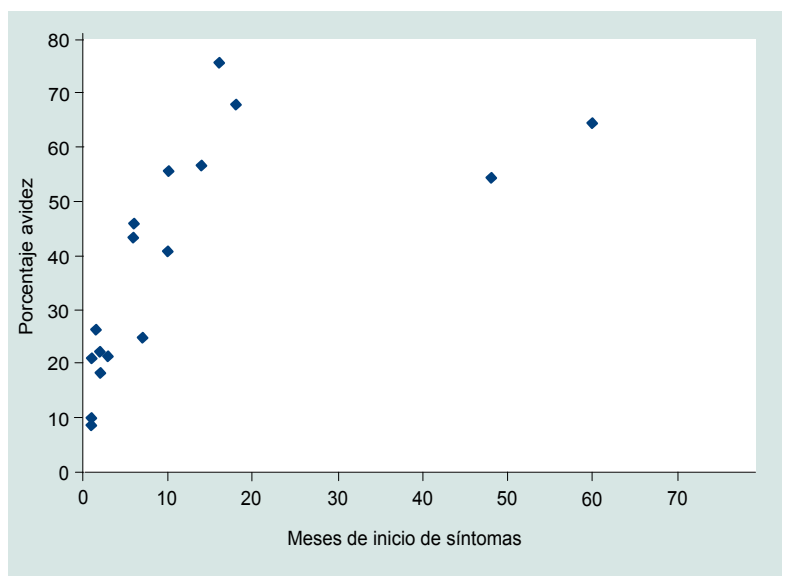


Figura 2. Curva receptor operador (ROC) IgG de avidez para Toxoplasma, con un punto de corte menor al 30\%, se obtiene una sensibilidad del $100 \%$ y una especificidad del $88 \%$.

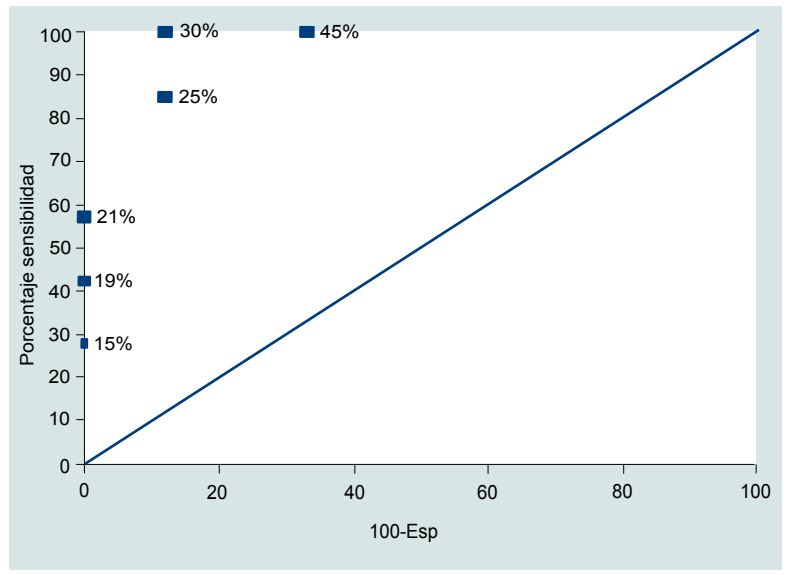

se usó la de marca Sigma (Ref: U1250). De manera interesante, en estos pacientes con fecha conocida de infección, la IgM fue positiva en todos hasta el mes 24 y la IgA fue positiva sólo en pacientes con menos de 4 meses de infección, aunque en uno de ellos la prueba fue negativa, es decir que hubo $87 \%$ de positividad en infecciones menores de 4 meses para la IgA (figura 3).

Figura 3. Evolución de los valores de absorbancia para la prueba ELISA IgM (cutoff 0,3) e IgA (cutoff 0,2) en 17 pacientes con fechas de infección conocida.

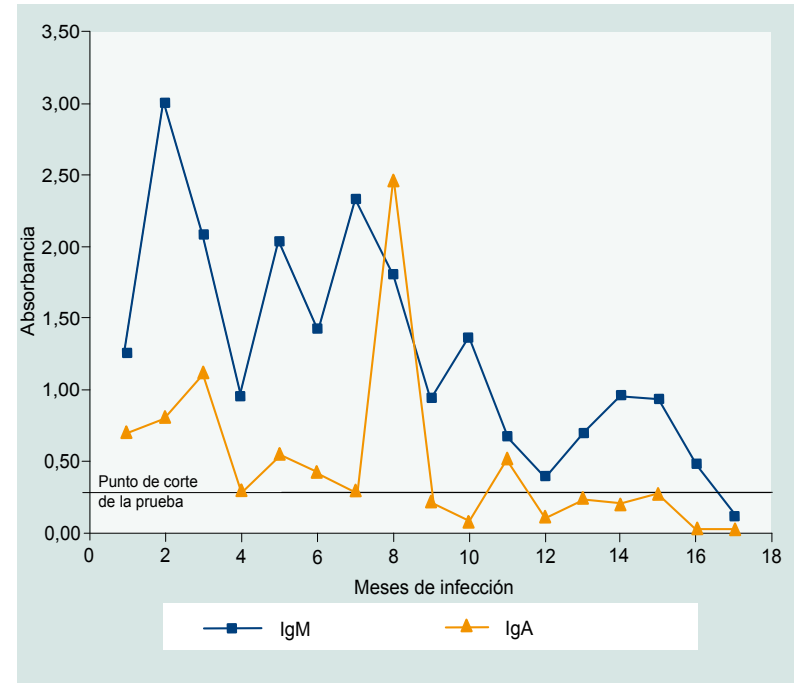

\section{Utilización de la prueba de IgG avidez en gestantes durante el control prenatal}

En un grupo de sueros de pacientes con resultados positivos para IgG y para los cuales se solicitó la prueba de avidez, se determinó si se está solicitando la prueba durante el primer trimestre de gestación, durante el cual los resultados de avidez pueden tener una utilidad clínica importante. Para esto se revisaron los datos de pacientes a quienes se les realizó prueba de avidez en el Centro de Investigaciones Biomédicas y se analizó la relación entre las semanas de gestación en las cuales se hicieron la prueba de avidez (figura 4). Se encontró que el 63\% tenía menos de 16 semanas (4 meses) al momento de la prueba, de ellas 3 tuvieron índices de avidez bajo (<30\%). Todas las cuatro pacientes con índices de avidez por debajo de 30\% fueron IgM positivas pero ELISA IgA negativas. El único caso en el cual las IgM e IgA fueron positivas tuvo un índice de avidez de $61 \%$ a las 12 semanas de gestación, indicando que la infección ocurrió 4 meses antes, es decir fue preconcepcional.

Figura 4. Gestantes que se realizaron la prueba IgG de avidez en el laboratorio del Centro de Investigaciones Biomédicas de la Universidad del Quindío, nótese que 3 gestantes tuvieron índices de avidez bajo (<30\%) en la semana 12 .

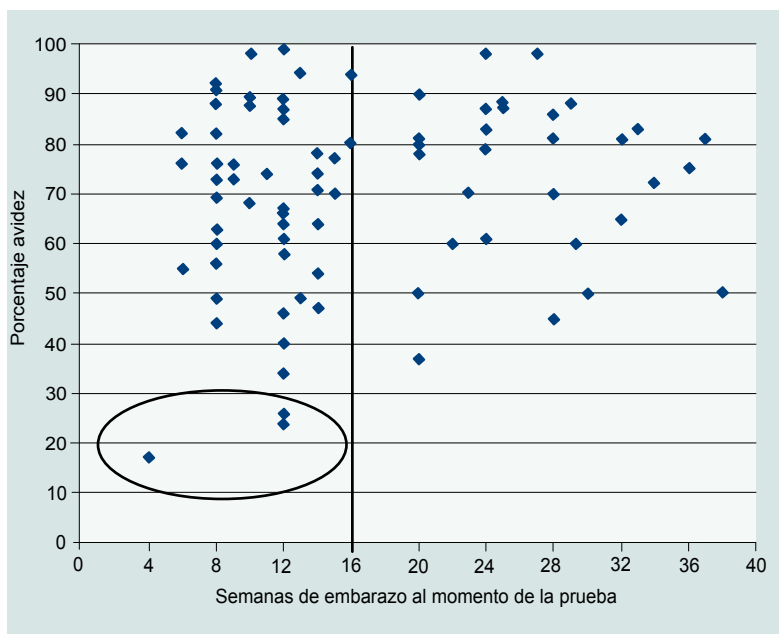




\section{DISCUSIÓN}

Se observó una relación entre la duración de la infección y los porcentajes de avidez en los sueros con fechas conocidas de infección. En nuestra prueba, un porcentaje de avidez menor a $30 \%$ indica infecciones adquiridas hace menos de 4 meses. Esto es un resultado idéntico para el punto de corte del estudio de Maekelt, et al. ${ }^{10}$ Sólo encontramos un caso con avidez menor de 30\% y evolución de 8 meses; esto apunta a una avidez baja persistente, fenómeno ya reportado en la literatura. ${ }^{8,11}$ El resultado de la curva ROC es también en todo similar a lo reportado en la literatura para esta prueba. ${ }^{8} \mathrm{Se}$ confirma la utilidad, como se preconiza en la Guía Nacional para Toxoplasmosis,${ }^{12}$ de realizar la avidez antes de la semana 16, así en un caso con IgM e IgA positivas el resultado de la avidez orienta a una infección preconcepcional con muy bajo riesgo de transmisión. Nuestra curva también mostró que la prueba ELISA IgA fue positiva sólo en infecciones menores de 4 meses y que algunos pacientes no desarrollan IgA en una primoinfección. Esto muestra que la prueba ELISA IgA es menos sensible pero más específica y confirma su utilidad y límites para la orientación diagnóstica. ${ }^{13}$ Igualmente, nuestros resultados ponen en evidencia el hecho de que, si se hacen pruebas de avidez luego de la semana 16 , las infecciones adquiridas al inicio de la gestación pueden no diagnosticarse. En el caso de los sueros analizados en nuestro laboratorio, se observa que la mayoría se hacen antes de la semana 16 (63\%) lo cual es un indicador positivo, pues se está solicitando la prueba tempranamente.

El presente trabajo corrobora la observación de otros autores de que la prueba de avidez ELISA para IgG anti-Toxoplasma puede ser una valiosa ayuda para distinguir mejor entre una infección por Toxoplasma reciente (menor de 4 meses) y una crónica. La confirmación de un diagnóstico de infección por Toxoplasma reciente es crucial para la clínica en la toma de decisión terapéutica precoz, especialmente en embarazadas con primoinfecciones e infecciones congénitas asintomáticas. La fortaleza de este estudio es que se utilizaron muestras con fechas conocidas de infección, las cuales son de difícil obtención; en nuestro caso se requirieron más de dos años para obtener este grupo de casos. La debilidad del estudio es el bajo número de muestras utilizadas, lo cual genera un intervalo de confianza amplio para el valor de sensibilidad obtenido.

\section{CONCLUSIONES}

Un punto de corte de 30\% para la prueba ELISA de avidez anti-Toxoplasma casera en el Centro de Investigaciones Biomédicas de la Universidad del Quindío, identifica infecciones de menos de 4 meses de adquirida.

\section{REFERENCIAS}

1. Nissapatorn V, Lee C, Kia F, Lleong C, Mahmud $\mathrm{R}$, Abdullah KA. Toxoplasmosis in HIV/AIDS patients: a current situation. Jpn J Infect Dis 2004; 57:160-5.

2. Wong SY, Remington JS. Toxoplasmosis in pregnancy. Clin Infect Dis 1994;18:853-62.

3. Montoya JG, Liesenfeld O. Toxoplasmosis. Lancet 2004;363:1965-76.

4. Remington JS, McLeod R, Thulliez P, Desmonts G. Toxoplasmosis. En: Remington JS, Wilson CB, Baker CJ. Klein JO. (eds.) Infectious diseases of the fetus and newborn infant. 6th ed. Philadelphia: WB Saunders; 2006. p. 917-1092.

5. Rosso F, Agudelo A, Isaza A, Montoya JG. Toxoplasmosis congénita: aspectos clínicos y epidemiológicos de la infección durante el embarazo. Colomb Med 2007;38:316-37.

6. Hedman K, Lappalainen M, Seppala I, Mäkelä O. Recent primary Toxoplasma infection indicated by a low avidity of specific IgG. J Infect Dis 1989; 159:736-9.

7. Remington JS, Thulliez P, Montoya JG. Recent developments for diagnosis of toxoplasmosis. J Clin Microbiol 2004;42:941-5.

8. Cozon GJ, Ferrandiz J, Nebhi H, Wallon M, Peyron F. Estimation of the avidity of immunoglobuling for routine diagnosis of chronic Toxoplasma gondii infection in pregnant women. J Clin Microbiol Infect Dis 1998;17:32-6. 
9. Camargo ME, Silva SM, Leser PG, Granato CH. Avidez de anticorpos IgG específicos como marcadores de infecçâo primaria recente pelo Toxoplasma gondii. Rev Inst Med Trop Sao Paulo 1991;33:213-8.

10. Maekelt A, Mauriello L, Diaz MP. Evaluación de la prueba ELISA-Avidez-IgG como inmunodiagnóstico serológico de la infección toxoplasmósica reciente. RFM 2000;23:149-56.

11. Montoya JG, Huffman HB, Remington JS. Evaluation of the immunoglobulin $\mathrm{G}$ avidity test for diagnosis of toxoplasmic lymphadenopathy. J Clin Microbiol 2004;42:4627-31.

12. Gómez JE, Ruiz B, Silva P, Beltrán S, Cortés J, Montoya J, et al. Guía de práctica clínica para toxoplasmosis durante el embarazo y toxoplasmosis congénita en Colombia. Infectio 2007;11:1-13.

13. Montoya MT, Gómez Marín JE, Loango N, Castaño JC, Marx C, Foudrinier F, et al. Utilidad de dos pruebas serológicas para IgA humana anti-toxoplasma como pruebas de referencia para toxoplasmosis materna reciente. Acta Med Colomb 1998;23:275-82.

Conflicto de intereses: los autores declaran no haber recibido salarios ni beneficios de parte de las empresas distribuidoras o fabricantes de los estuches utilizados en este estudio. 